\title{
Two-photon photochemical long-period grating fabrication in hydrogenated photonic crystal fiber
}

\author{
Gilberto Brambilla (1), Philip St. J. Russell (2), Andrei Fotiadi (3), Stephen A. Slattery (4), Thomas Ernst (4), \\ David N. Nikogosyan (4) \\ 1 : Optoelectronics Research Centre, University of Southampton, UK \\ 2 : Max Planck Research Group, University of Erlangen-Nuremberg, Germany \\ 3 : Faculté Polytechnique de Mons, Mons, Belgium \\ 4 : Physics Department, National University of Ireland, University College Cork, Cork, Ireland, niko@phys.ucc.ie
}

\begin{abstract}
We report on the photochemical fabrication of a long-period grating in photonic crystal fiber. The characteristic fluence value for inscription is an order of magnitude less than that for standard telecom fiber.
\end{abstract}

\begin{abstract}
Introduction
Long-period fiber grating (LPFG) fabrication in a photonic crystal fiber (PCF) has been known since $2002^{1}$. Non-photochemical inscription techniques are commonly used to modify the refractive index in the fiber cladding either by heating (using $\mathrm{CO}_{2}$ laser light $^{1,2}$ or an electric arc discharge ${ }^{3,4,5}$ ) or by applying mechanical pressure ${ }^{6}$. In this paper we report on the photochemical recording of LPFGs in a PCF, which is based on two-photon absorption (TPA) of highintensity femtosecond $264 \mathrm{~nm}$ pulses in pure fused silica.
\end{abstract}

\section{Experiment}

We used an endlessly single mode PCF (ESM-12-01) from Crystal Fibre $\mathrm{A} / \mathrm{S}$ and for comparison the standard Corning telecom fiber SMF-28 (supplied by Elliot Scientific). ESM-12-01 has a $12 \mu \mathrm{m}$ core diameter surrounded by 4 rings of holes (hole diameter is $3.7 \mu \mathrm{m}$, hole pitch is $8 \mu \mathrm{m}$, number of holes is 54), and its outside diameter is $125 \mu \mathrm{m}$. SMF28 has a core diameter of $8.2 \mu \mathrm{m}$ and a cladding diameter of $125 \mu \mathrm{m}$. Both fibers were sensitized under similar conditions (in a hydrogen atmosphere at 150 bar, at $70{ }^{\circ} \mathrm{C}$, for 2 weeks). To decrease the rate of hydrogen out-diffusion from the PCF, it was pigtailed to two $0.5 \mathrm{~m}$ long pieces of SMF-28 fiber by splicing before hydrogenation.

For LPFG inscription, we applied femtosecond 264 $\mathrm{nm}$ laser pulses with an incident irradiation intensity in the $300-400 \mathrm{GW} / \mathrm{cm}^{2}$ range. The fibre was irradiated according to the method described earlier ${ }^{7,8}$. The irradiated fiber was exposed point by point with a period of $500 \mu \mathrm{m}$. The length of the LPFG was $1 \mathrm{~cm}$.

\section{Results}

Figures 1(a), 1(b) and 1(c) present the typical spectrum of transmission loss, and the peak wavelength and amplitude dependencies versus the incident fluence, respectively, for an LPFG inscribed in the PCF. Figures 2(a), 2(b) and 2(c) present the corresponding dependencies for an LPFG inscribed in the SMF-28 fiber.

The LPFG spectrum created in the PCF shows very strong resonance peaks with up to $\sim 20 \mathrm{~dB}$ grating strength (Fig. 1(a)). As the band-gap energy value of pure fused silica is about $9.3 \mathrm{eV}$, this band-gap could be bridged only by two $264 \mathrm{~nm}$ light quanta with an energy of $4.7 \mathrm{eV}$, which is in line with the recent experiment on fiber Bragg grating (FBG) inscription in a PCF using high-intensity $267 \mathrm{~nm}$ pulses ${ }^{9}$. The loss spectrum also demonstrates an excellent LPFG quality (regular form of the peaks and absence of outband losses). All the transmission loss peaks are shifting monotonically towards the longer wavelengths with the increase in incident fluence (Fig. 1 (b)). From Fig. 1(c) it follows that at an incidence fluence of 10 $\mathrm{J} / \mathrm{cm}^{2}$, peak $A$ reaches its maximum (coupling factor is equal to $\pi / 2$ ). Such an effect has never before been demonstrated in a PCF. But the most striking feature of the data presented in Fig. 1 (c) is the extremely low value of fluence necessary for the recording of an LPFG in a hydrogenated PCF.

Indeed, to fabricate a FBG in a hydrogenated PCF with a reflection peak of $7 \mathrm{~dB}^{9}$, one needs a huge fluence value of $70 \mathrm{~kJ} / \mathrm{cm}^{2}$. In our experiments with FBG inscription in $\mathrm{H}_{2}$-loaded SMF-28, a fluence value of $1.2 \mathrm{~kJ} / \mathrm{cm}^{2}$ was sufficient to record a reflection peak of more than $45 \mathrm{~dB}^{10}$, meaning that Bragg grating fabrication in a PCF requires two orders more fluence. In contrary, for LPFG fabrication in PCF, only $10 \mathrm{~J} / \mathrm{cm}^{2}$ fluence is enough to record a $\sim 20 \mathrm{~dB}$ peak, in comparison with $\sim 120(160) \mathrm{J} / \mathrm{cm}^{2}$ fluence value necessary for the inscription of the $\sim 24 \mathrm{~dB} \mathrm{~K}$ (or L) peak in SMF-28 (Figs. 2(a), 2(c)).

Both these effects, the difficulty in writing a FBG in PCF and the easiness of recording an LPFG, could be explained by a quick redistribution of hydrogen in a PCF before inscription. Probably, the presence of the holes effectively reduces the concentration of hydrogen in the core and simultaneously increases it in the cladding. The ring-like distribution of hydrogen in the PCF cladding creates a similar distribution of refractive index change. 

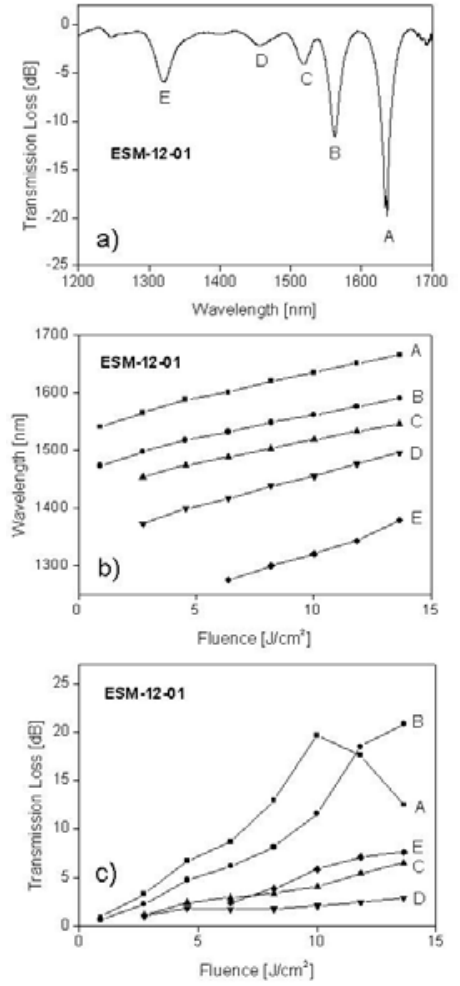

Fig. 1. The experimental graphs related to LPFG inscribed in a PCF: (a) transmission loss spectrum recorded with an irradiation intensity of $300 \mathrm{GW} / \mathrm{cm}^{2}$ and a total incident fluence of $10 \mathrm{~J} / \mathrm{cm}^{2}$; (b) shift of wavelengths corresponding to the transmission loss peaks $A, B, C, D$, and $E$ (designated in the spectrum above) versus the total incident fluence; (c) transmission loss amplitudes for the peaks $A, B, C, D$, and $E$ versus the total incident fluence.

This statement is validated by the wavelength position of similar transmission loss peaks in LPFGs written in PCF and SMF-28. Another factor increasing the efficiency of LPFG fabrication in a PCF is connected with a higher uniformity of PCF illumination, which is due to the presence of non-absorbing holes and the absence of a highly absorbing fiber core (by the TPA mechanism ${ }^{11}$ ). Indeed, a simple estimate using the TPA coefficient value for fused silica ${ }^{11}$ gives a value of only $5 \%$ for the absorbed energy inside the PCF. As a result, the irradiation intensity is more spatially uniform and doesn't drop quickly, bringing about an overall higher yield of TPA-formed refractive index changes. The third factor improving the efficiency of LPFG inscription is the larger core diameter in the PCF in comparison to that in SMF-28 causing a stronger overlap between the core and cladding modes in the PCF.

\section{Conclusion}

The TPA-based high-intensity $264 \mathrm{~nm}$ femtosecond
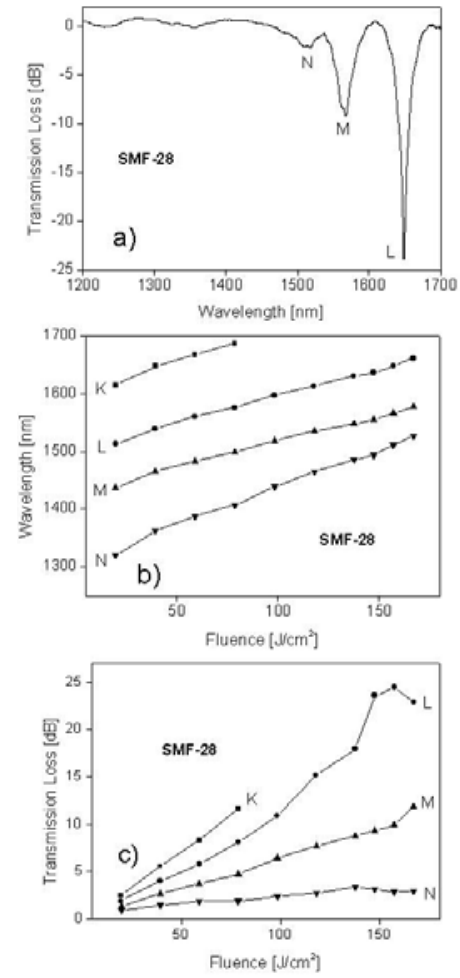

Fig. 2. The experimental graphs related to LPFG inscribed in SMF-28: (a) transmission loss spectrum recorded with an irradiation intensity of $310 \mathrm{GW} / \mathrm{cm}^{2}$ and a total incident fluence of $157 \mathrm{~J} / \mathrm{cm}^{2}$, the peak $\mathrm{K}$ at this fluence value moved beyond $1700 \mathrm{~nm}$; (b) shift of wavelengths corresponding to the transmission loss peaks $K, L, M$, and $N$ versus the total incident fluence; (c) transmission loss amplitudes for the peaks $K, L, M$, and $N$ versus the total incident fluence.

approach allows us to fabricate LPFGs in PCF using very low fluences in comparison with the inscription in a standard telecom fiber under similar irradiation conditions.

\section{References}

1. G. Kakarantzas et al Opt. Lett., 27 (2002), 101

2. Y. Zhu et al Opt. Lett., 28 (2003), 2467

3. G. Humbert et al Electron. Lett., 39 (2003), 349

4. K. Morishita et al J. Lightwave Technol., 22 (2004), 625

5. H. Dobb et al Electron. Lett., 40 (2004), 657

6. J.H. Lim et al Opt. Lett., 29 (2004), 331

7. A.I. Kalachev et al Opt. Commun., 246 (2005), 107; Errata, Opt. Commun. 251 (2005), 229

8. A.I. Kalachev et al J. Lightwave Technol., 23 (2005), 2568

9. L.B. Fu et al Electron. Lett., 41 (2005), 638

10. S.A. Slattery et al J. Opt. Soc. Am. B, 22 (2005),

354; Errata, J. Opt. Soc. Am. B, 22 (2005), 1143

11. A. Dragomir et al Appl. Phys. Lett., 80 (2002), 11 